\title{
HSP47 is associated with the prognosis of laryngeal squamous cell carcinoma by inhibiting cell viability and invasion and promoting apoptosis
}

\author{
XIAOXIAO SONG ${ }^{1 *}$, ZHISU LIAO $^{1 *}$, CHUNCHUN ZHOU $^{1}$, RENYU LIN $^{1}$, JIANGLONG LU $^{2}$, LIN CAI $^{2}$, \\ XIAOHUA TAN ${ }^{3}$, WENJIAN ZENG ${ }^{1}$, XIANGHE LU ${ }^{2}$, WEIMING ZHENG ${ }^{2}$, \\ JIANFU CHEN ${ }^{1}$ and ZHIPENG SU ${ }^{2}$ \\ Departments of ${ }^{1}$ Otolaryngology and ${ }^{2}$ Neurosurgery, The First Affiliated Hospital of Wenzhou Medical University; \\ ${ }^{3}$ School of Pharmaceutical Sciences, Key Laboratory of Biotechnology and Pharmaceutical Engineering, \\ Wenzhou Medical University, Wenzhou, Zhejiang 325000, P.R. China
}

Received February 24, 2016; Accepted July 24, 2017

DOI: $10.3892 /$ or.2017.5893

\begin{abstract}
Heat shock protein 47 (HSP47) is a $47 \mathrm{kDa}$ collagen binding protein that has a close relationship with the development and progression of tumours. However, little is known concerning the expression profile of HSP47 in laryngeal squamous cell carcinoma (LSCC) patients and there is still insufficient data concerning the underlying mechanisms. The aim of the present study was to explore the expression of HSP47 in LSCC and provide an overview of its association with tumourigenicity and clinical prognosis. The expression of HSP47 in LSCC and adjacent non-cancerous laryngeal tissues was assessed via western blotting and immunohistochemical studies. The prognostic significance of HSP47 expression was analysed using a Kaplan-Meier survival curve. To investigate the influence of HSP47 on the viability, invasion and apoptosis
\end{abstract}

Correspondence to: Dr Zhipeng Su, Department of Neurosurgery, The First Affiliated Hospital of Wenzhou Medical University, 3 Fuxue Road, Wenzhou, Zhejiang 325000, P.R. China

E-mail: drsuzhipeng@163.com

Dr Jianfu Chen, Department of Otolaryngology, The First Affiliated Hospital of Wenzhou Medical University, 3 Fuxue Road, Wenzhou, Zhejiang 325000, P.R. China

E-mail: chenjf_123@163.com

*Contributed equally

Abbreviations: LSCC, laryngeal squamous cell carcinoma; HSP47, heat shock protein 47; FBS, fetal bovine serum; BCA, bicinchoninic acid; PVDF, polyvinylidene fluoride; siRNA, small interfering RNA; CCK-8, Cell Counting Kit-8; PBS, phosphate-buffered saline; FITC, fluorescein isothiocyanate; PI, propidium iodide; FCM, flow cytometry; OS, overall survival; HSPs, heat shock proteins; HESCC, human esophageal squamous cell carcinoma

Key words: HSP47, laryngeal squamous cell carcinoma, prognosis, cisplatin, proliferation, invasion, apoptosis of a LSCC cell line, we performed an in vitro analysis with plasmid vectors and small interfering RNA (siRNA). Our results showed that HSP47 protein expression in the LSCC tissues was markedly decreased compared to that noted in the adjacent non-cancerous tissues, and low expression of HSP47 was correlated with poor prognosis in LSCC patients. Upregulation of HSP47 via plasmid vectors inhibited the proliferation, reduced the invasive ability, increased the sensitivity to cisplatin chemotherapy, promoted apoptosis, and induced the G1 phase arrest of LSCC cells in vitro. The expression of apoptosis-regulating proteins was also altered when HSP47 was upregulated, involving increased expression of cleaved caspase-7/-8/-9, PARP, and Bax and decreased expression of $\mathrm{Bcl}-2$. Our present data suggest that HSP47 is an important prognostic factor and an attractive therapeutic target in LSCC due to its influence on the biological behaviour of LSCC cells.

\section{Introduction}

Laryngeal squamous cell carcinoma (LSCC) is the second most common malignant cancer type of the head and neck $(1,2)$. Approximately 650,000 cases are newly diagnosed and more than 350,000 deaths are attributed to this disease every year in the US $(1,3)$. Due to high exposure to tobacco and alcohol risk factors, middle-aged and elderly men often suffer from this disease, and its incidence has been rising in recent years (4). Therapeutic measures for LSCC include surgery, chemotherapy or radiotherapy and comprehensive surgery, radiotherapy and chemotherapy (5-8). The clinical prognosis of this disease, however, is poor, and LSCC continues to be one of the major causes of cancer-related deaths (9). An epidemiological survey (2) indicated that the clinical outcome of LSCC has not obviously improved over the past 20 years, despite considerable improvements in technologies related to LSCC detection and diagnosis. Therefore, it is necessary to develop valuable biomarkers to identify patients with a poor prognosis and the risk factors for recurrence, which can serve as a guide for determining the surgical approach and the combination treatment scheme. 
Heat shock protein 47 (HSP47), also known as colligin-2, is a type of endoplasmic reticulum resident collagen protein and specific molecular chaperone, which is essential for the synthesis and secretion of procollagens (10). HSP47 is a product of the $C B P 2$ gene, located at chromosome $11 \mathrm{q} 13.5$ (11), which is a region frequently involved in the biological behaviour of malignant tumours, including oral tongue cancer, nasopharyngeal and esophageal squamous cell carcinoma, lung diseases, pancreatic ductal adenocarcinoma, cervical squamous cell and ulcerative colitis-associated carcinoma (12-17). As a classical serpin, HSP47 has been proven to exert inhibitory effects on tumour proliferation, invasion and motility $(18,19)$. However, the profile and role of HSP47 expression in LSCC patients and the potential mechanisms underlying the generation, development and metastasis of tumours have not been fully explored.

In the present study, we examined the HSP47 expression and its prognostic significance in LSCC patients. Moreover, the effects of HSP47 on cell viability, invasion, chemotherapeutic sensitivity and apoptosis were evaluated in vitro. We further explored the potential apoptotic mechanisms of HSP47, which may be related to the regulation of PARP and cleaved caspase-7/-8/-9. We conclude that HSP47 may act as an important prognostic biomarker and an attractive therapeutic target in LSCC.

\section{Materials and methods}

LSCC tissue collection. A total of 62 LSCC patients who received no radiotherapy or chemotherapy and who underwent surgery between 2011 and 2015 at the First Affiliated Hospital of Wenzhou Medical University were examined in this retrospective research. All samples were from 61 males and 1 female with ages ranging from 43 to 79 years (mean, 64.5 years). The present study was approved by the Clinical Medicine Ethics Committee of The First Affiliated Hospital of Wenzhou Medical University, and consent for enrollment in the study was provided by the patients. The study protocol was carried out according to the principles of the Helsinki Declaration. In total, 50 formalin-fixed, paraffin-embedded tumour sections were obtained from the pathology department for immunohistochemical analysis, and 7 samples of histologically normal laryngeal tissues surrounding the tumours were treated as control tissues. Another 12 pairs of fresh-frozen LSCC tissues which were used for western blot analysis, including cancerous and matched adjacent non-cancerous tissues from the same patients, were maintained at $-80^{\circ} \mathrm{C}$. Patient follow-up was performed via telephone calls until October 2016. The outcome event was defined according to the date of death.

Cell culture and reagents. The human LSCC, Hep-2 cell line, was obtained from the Type Culture Collection of the Chinese Academy of Sciences (Shanghai, China). In brief, cells were cultivated in a complete medium containing RPMI-1640 medium, 10\% fetal bovine serum (FBS) (both from HyClone, Logan, UT, USA) and $1 \%$ antibiotics $(100 \mathrm{U} / \mathrm{ml}$ penicillin/streptomycin) in a humidified incubator at $37^{\circ} \mathrm{C}$ with $5 \%$ $\mathrm{CO}_{2}$. Cisplatin was obtained from Qilu Pharmaceutical Co., Ltd. (Shandong, China).
Western blot analysis. Briefly, total protein was extracted and the concentration was measured with a bicinchoninic acid (BCA; Beyotime, Jiangsu, China) protein assay kit. For each sample, $\sim 30-50 \mu \mathrm{g}$ of protein were run on a $10 \%$ polyacrylamide gel, and then transferred to polyvinylidene fluoride (PVDF) membranes. The membranes were immunoblotted with the primary antibodies, followed by the secondary HRP-conjugated antibody. Immunoreactive proteins were visualized using ECL western blot detection kit (Advansta, Menlo Park, CA, USA) and quantified employing Image Lab software (Bio-Rad, Hercules, CA, USA). Information concerning the antibodies was as follows: rabbit anti-GAPDH polyclonal antibody (ab-p-r001; 1:1,000; Xianzhi, Zhejiang, China); rabbit anti-HSP47 monoclonal antibody (ab109117; 1:3,000; Abcam, Cambridge, UK); rabbit anti-Bcl-2 monoclonal antibody (\#4223; 1:1,000); rabbit anti-Bax monoclonal antibody (\#5023; 1:1,000); rabbit anti-PARP monoclonal antibody (\#9532; 1:1,000); rabbit anti-cleaved caspase-7 monoclonal antibody (\#8438; 1:1,000); rabbit anti-cleaved caspase-8 monoclonal antibody (\#9496; 1:1,000); rabbit anti-cleaved caspase-9 monoclonal antibody (\#9505; 1:1,000) (all from Cell Signaling Technology, Danvers, MA, USA).

Immunohistochemistry. Fifty formalin-fixed, paraffinembedded tumour sections and 7 control tissues were stained with HSP47-specific antibody raised against the amino acid sequence of human HSP47 (ab109117; 1:300; Abcam). Briefly, the sections $(5-\mu \mathrm{m})$ were deparaffinized in dimethylbenzene, dehydrated in graded alcohols, subjected to peroxidase blocking and then retrieved with citrate buffer. The sections were immunoblotted with the anti-HSP47 antibody at a dilution of 1:300 overnight at $4^{\circ} \mathrm{C}$, followed by incubation with the secondary HRP-conjugated antibody. The sections were then counterstained with haematoxylin and observed under a biological imaging microscope (BX53; Olympus, Tokyo, Japan), which also was used to obtain images. All of the immunohistochemically stained sections were evaluated via a double-blind semi-quantitative analysis to avoid artificial errors. Cytoplasmic staining was regarded as positive. In total, 10 high-power fields (magnification, x200) within the stained tumour cytoplasm were selected to analyse the expression of HSP47. In the present study, to evaluate prognosis, we divided the extent of staining into 2 groups: a high-score group (staining extent $\geq 50 \%$ ) and a low-score group (staining extent $<50 \%$ ). These cut-off values have been used in past studies $(20,21)$.

Quantitative RT-PCR. According to the protocol of the manufacturer, total RNA from the Hep-2 cells was extracted using Trizol (Invitrogen, Carlsbad, CA, USA). The first-strand cDNAs were synthesized using a reverse transcription kit (Takara, Dojindo, Japan). The synthesized cDNA $(2 \mu \mathrm{l})$ was amplified (final volume, $20 \mu \mathrm{l}$ ) using a SYBR-Green PCR kit (Takara) and loaded on the ABI 7500 sequence detection system (Applied Biosystems, Foster City, CA, USA). DNA amplification conditions were as follows: $95^{\circ} \mathrm{C}$ for $30 \mathrm{sec}$ and subsequent 40 cycles, $95^{\circ} \mathrm{C}$ for $5 \mathrm{sec}, 60^{\circ} \mathrm{C}$ for $34 \mathrm{sec}$. The primer information was as follows: HSP47 (human forward) 5'-CACCGCCTTTGAGTTGGACAC-3' and HSP47 (human reverse) 5'-GGCGCCCAATGAATAGCAG-3'; GAPDH 
(human forward) 5'-ATGAGCCCCAGCCTTCTCCAT-3' and GAPDH (human reverse) 5'-GGTCGGAGTCAACGGAT TTG-3'. The $2^{-\Delta \Delta C t}$ analysis was used to calculate gene expression levels.

Gene overexpression and silencing. According to the reagent protocol, the cells were transiently transfected with plasmid DNA or small interfering RNA (siRNA) using Lipofectamine 3000 (Invitrogen). In regards to the plasmid transfection, Lipofectamine 3000 was diluted in Opti-MEM medium after the cells were seeded at $80-90 \%$ confluence. Meanwhile plasmid DNA and P3000 reagent were diluted in Opti-MEM medium. Each was incubated for $5 \mathrm{~min}$, respectively, and then mixed together for $20 \mathrm{~min}$. Finally, the DNA-lipid complex was added to the cells. Gene transfection of siRNA was the same, except for the addition of P3000 reagent when diluting the siRNA. Quantitative RT-PCR and western blotting were used to compare transfection efficiency. (pCDH)-HSP47, (pCDH)-control, siHSP47 and siControl were all obtained from GenePharma (Shanghai, China). Information regarding the siRNAs was as follows: siHSP47, 5'-GCAGCA AGCAGCACUACAATT-3' and siControl, 5'-TTCTCCGAA CGTGTCACGTTT-3'.

Cell viability assay. Cell viability was assessed using Cell Counting Kit-8 (CCK-8; Dojindo, Kumamoto, Japan). After transfection for $24 \mathrm{~h}$, the cells were plated into 96-well plates at a density of $1 \times 10^{3}$ cells/well, and then subjected to cell proliferation assay by CCK-8 at 24, 48, 72 and $96 \mathrm{~h}$. For chemosensitivity analysis, the cells were transfected with (pCDH)-HSP47 or siHSP47 for $24 \mathrm{~h}$, and then plated into 96 -well plates at a density of $4 \times 10^{3}$ cells/well. The cells were subsequently treated with cisplatin $(9 \mu \mathrm{M} / \mathrm{l})$ for $24-96 \mathrm{~h}$, and subjected to cell proliferation assay by CCK-8 at 24, 48 , 72 and 96 h. Each group contained 3 parallel wells. CCK-8 $(10 \mu \mathrm{l})$ was added to the cell culture medium $(90 \mu \mathrm{l})$ according to the manufacturer's instructions. The cells were subsequently cultured for $2 \mathrm{~h}$ in an incubator at $37^{\circ} \mathrm{C}$ and the absorbance was measured at $450 \mathrm{~nm}$.

Transwell invasion assay. The cells on the lower surface of the membrane were quantified to assess the invasive potential. As follows, Matrigel matrix gel (BD Biosciences, San Jose, CA, USA) was diluted with serum-free culture medium at $1: 8$, for coating of the 24-well transwell chambers (Corning Costar, USA) which were placed in an incubator at $37^{\circ} \mathrm{C}$ overnight. The upper chamber was filled with the transfected cells $\left(1 \times 10^{5}\right.$ with $200 \mu \mathrm{l}$ of serum-free culture medium), and meanwhile the lower chamber was filled with $600 \mu \mathrm{l}$ of complete medium that contained $10 \%$ FBS. Following $18 \mathrm{~h}$ of cultivation, the cells in the upper chamber were wiped off with a cotton swab gently, and the invading cells that adhered to the lower membrane were fixed in paraformaldehyde for $15 \mathrm{~min}$, followed by crystal violet staining for $1 \mathrm{~h}$.

Wound-healing assay. The relative distance of Hep-2 cells moving into a wounded space was used to evaluate cell migration ability. Briefly, cells $\left(5 \times 10^{5}\right)$ were plated into 6 -well plates in serum-free medium for $24 \mathrm{~h}$. When achieving a monolayer, the confluent monolayers were scratched using a $10 \mu \mathrm{l}$ pipette tip and the floating cells were removed by phosphate-buffered saline (PBS). Finally, fresh culture medium was added to the wells. Phase contrast microscopy was performed to measure the relative distance of the wound.

Apoptosis assay and cell cycle analysis. Cells $\left(4 \times 10^{5}\right)$ were seeded into 6-well plates and transfected with the plasmid. After $48 \mathrm{~h}$ of culture, the cells were trypsinized, washed twice with PBS, and then either incubated with fluorescein isothiocyanate (FITC)-Annexin V/propidium iodide (PI) or only stained with PI (both from MultiSciences Biotech Co., Ltd., Zhejiang, China) in the dark according to the reagent protocol. A flow cytometer (FCM; Becton-Dickinson, San Jose, CA, USA) was used to calculated the percentages of apoptotic and necrotic cells and the DNA content.

Statistical analysis. All analyses were carried out using SPSS 16.0 software (SPSS, Inc., Chicago, IL, USA). Results are presented as mean values $\pm \mathrm{SD}$. The distinct expression of HSP47 protein between tumour tissues and controls was examined using paired-sample t-tests. The difference in overall survival (OS) between 2 groups was detected by the long-rank test. The prognostic value of HSP47 protein expression in LSCC patients was analysed by Kaplan-Meier survival curve. Tumour cell viability and invasion assays were assessed using independent $\mathrm{t}$-tests. $\mathrm{P}<0.05$ was considered to indicate a statistical significant result.

\section{Results}

Low expression of HSP47 protein is associated with a poor LSCC patient prognosis. To explore the expression of HSP47 in LSCC, we quantified the protein levels of HSP47 in matched cancerous and adjacent non-cancerous tissues from 12 LSCC patients by immunoblot analysis. As shown in Fig. 1A and B, in contrast to the controls, the level of HSP47 was significantly decreased in the tumour tissues $(\mathrm{P}<0.05)$. Furthermore, immunohistochemistry was performed on 50 formalin-fixed, paraffin-embedded LSCC and 7 adjacent normal laryngeal tissues. Based on the histopathologic grade, we classified the samples into 4 groups: normal, high differentiation, medium differentiation and low differentiation. As shown in Fig. 1C, the expression of HSP47 protein in the normal control tissues was obviously higher than that in the LSCC tissues with low to high differentiation, and the HSP47 protein was mainly localized in the cytoplasm. Moreover, the average immunohistochemical scores for HSP47 expression were observed to decrease as the tumour pathological grade of LSCC increased (Fig. 1D). Consistent with the results of the western blotting, LSCC tissues showed lower expression of HSP47 protein compared with that noted in the adjacent normal laryngeal tissues (Fig. $1 \mathrm{E} ; 71.0 \pm 7.1$ vs. $45.7 \pm 4.0 \%$, respectively; $\mathrm{P}<0.001)$.

We used a Kaplan-Meier survival curve to analyse the prognostic value of HSP47 protein expression in LSCC patients. We divided patients according to HSP47 protein expression levels into a low-score group (32.8 $22.4 ; 95 \% \mathrm{CI}, 27.9-37.7)$ and a high-score group (70.0 $\pm 2.4 ; 95 \% \mathrm{CI}, 65.0-85.0)$. As shown in Fig. 1F, the low-score group had a shorter OS time compared with the high-score group. The median OS time in the low- 
A

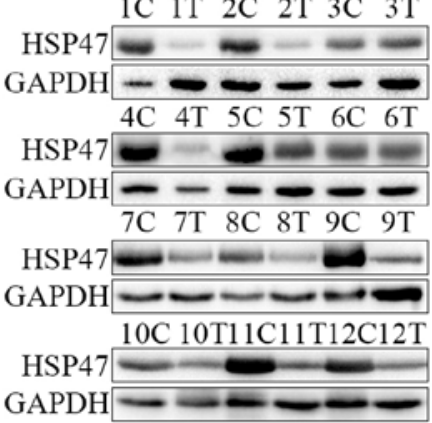

$\mathrm{D}$

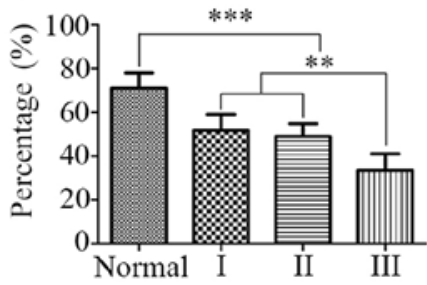

B

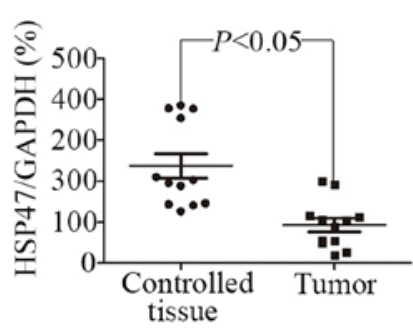

E

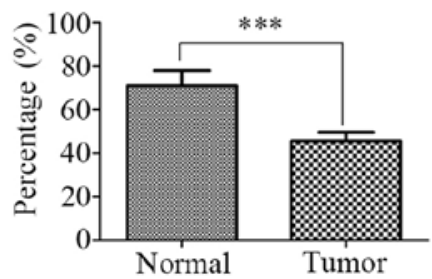

$\mathrm{C}$

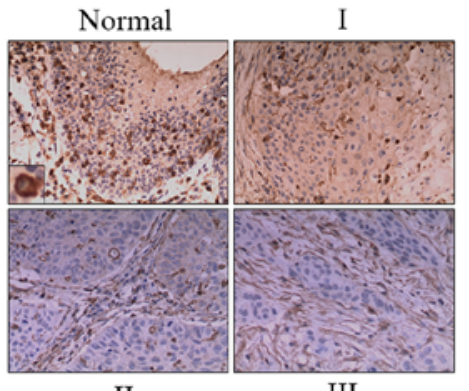

II

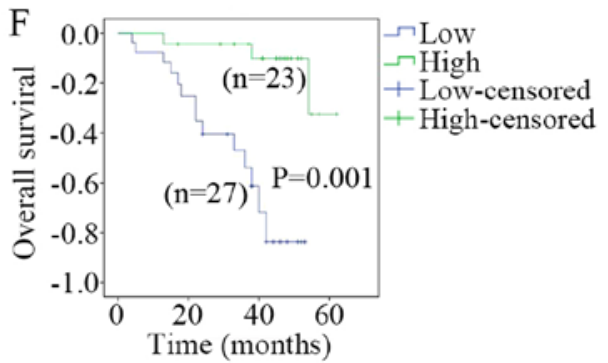

Figure 1. HSP47 exhibits low expression in LSCC tissues and is associated with improved prognosis in LSCC patients. (A and B) Western blot analysis indicated that HSP47 protein expression was obviously decreased in LSCC tissues compared to that noted in the matched adjacent non-cancerous tissues. C, adjacent non-cancerous tissues; T, LSCC tissues. (C) Immunohistochemical staining showed that the expression of HSP47 protein in normal control tissues was obviously higher than that in the LSCC tissues with low to high differentiation. HSP47 protein is expressed in the cytoplasm (C, left panel). I, high differentiation; II, medium differentiation; III, low differentiation. (D) The average immunohistochemical scores for HSP47 expression were decreased as the tumour pathological grade of LSCC was increased. (E) HSP47 protein expression was significantly decreased in LSCC tissues compared to matched adjacent non-cancerous tissues. (F) Kaplan-Meier survival curve showed that the low-score group had a shorter OS time compared with the high-score group; ${ }^{* *} \mathrm{P}<0.01$; **** $\mathrm{P}<0.001$

A

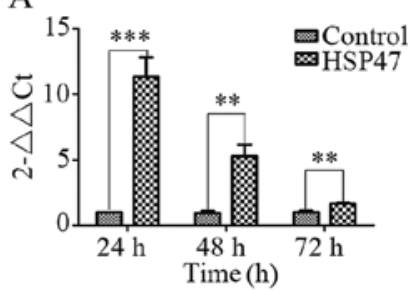

$\mathrm{D}$

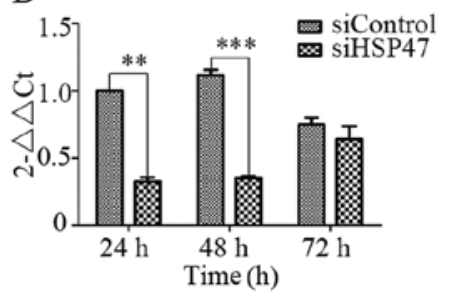

B

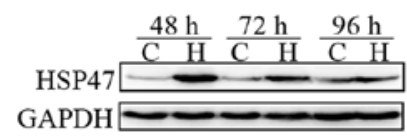

E

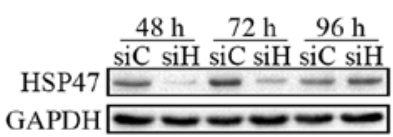

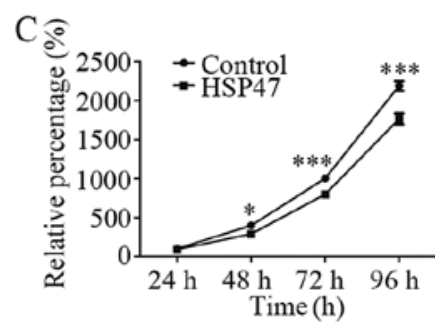

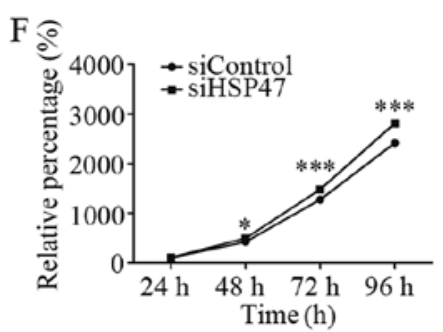

Figure 2. Upregulation of HSP47 inhibits Hep-2 cell proliferation. (A) Quantitative RT-PCR and (B) western blot analysis showed a marked increase in HSP47 expression after Hep-2 cell transfection with (pCDH)-HSP47. C, control; H, HSP47. (C) HSP47 upregulation via plasmid vectors inhibited cell proliferation in the Hep-2 cells. The cells were transfected with (pCDH)-HSP47 or siHSP47 for $24 \mathrm{~h}$, and then subjected to cell proliferation assay by CCK- 8 at 24,48 , 72 and 96 h. (D) Quantitative RT-PCR and (E) western blot analysis showed a significant decrease in HSP47 expression after Hep-2 cell transfection with siHSP47. siC, siControl; siH, siHSP47. (F) Knockdown of HSP47 enhanced the proliferative ability of Hep-2 cells; ${ }^{*} \mathrm{P}<0.05 ;{ }^{* * *} \mathrm{P}<0.01 ;{ }^{* * *} \mathrm{P}<0.001$.

score group was $38 \pm 2.5$ months, while in the high-score group was $46 \pm 2.9$ months $(\mathrm{P}=0.001)$.

Taken together, these results indicate that HSP47 may be an important prognostic factor for LSCC patients.

HSP47 overexpression inhibits the growth of Hep-2 cells. To analyze the effects of HSP47 on Hep-2 cells, cell proliferation analysis was performed after transfection with either a plasmid encoding HSP-47 [(pCDH)-HSP47)] or HSP47-specific siRNA (siHSP47) to upregulate or knockdown HSP47 levels in Hep-2 cells. As shown in Fig. 2A, (pCDH)-HSP47-transfected cells showed a marked increase in HSP47 mRNA expression as confirmed by real-time RT-PCR, while siHSP47-transfected cells exhibited a significant decrease (Fig. 2D). After 
A

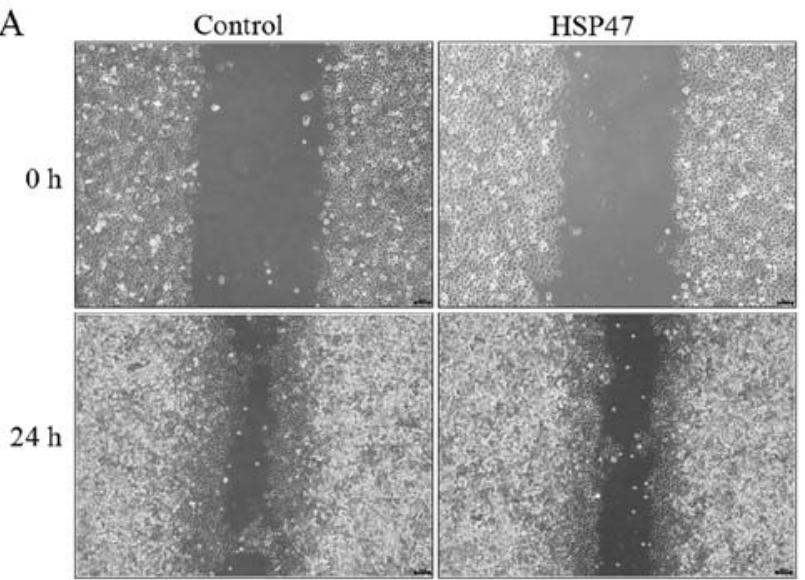

$\mathrm{C}$
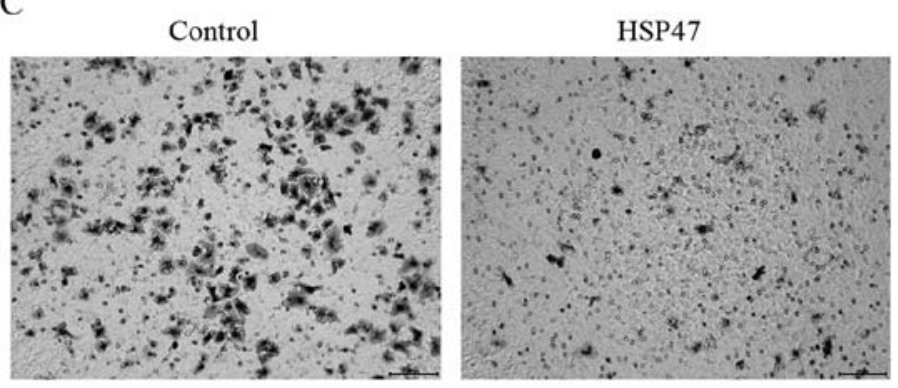

B
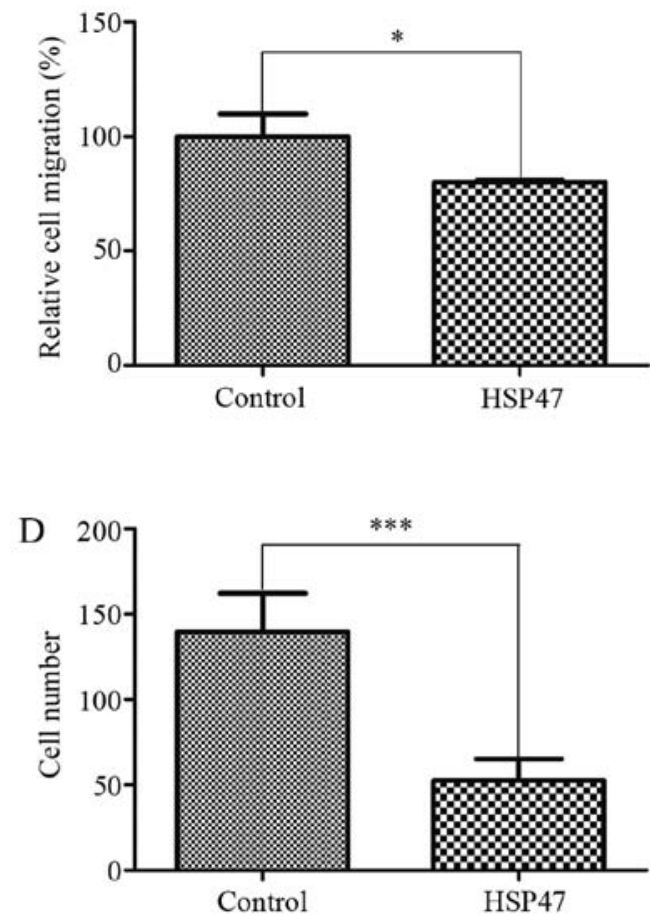

Figure 3. HSP47 upregulation suppresses Hep-2 cell migration and invasion abilities. (A and B) Wound-healing assay showed that HSP47 upregulation via plasmid vectors markedly inhibited wound-healing migration in the Hep-2 cells. (C and D) Transwell invasion assay showed that HSP47 overexpression via plasmid vectors notably suppressed invasion ability of the Hep-2 cells; ${ }^{*} \mathrm{P}<0.05,{ }^{* * *} \mathrm{P}<0.001$.
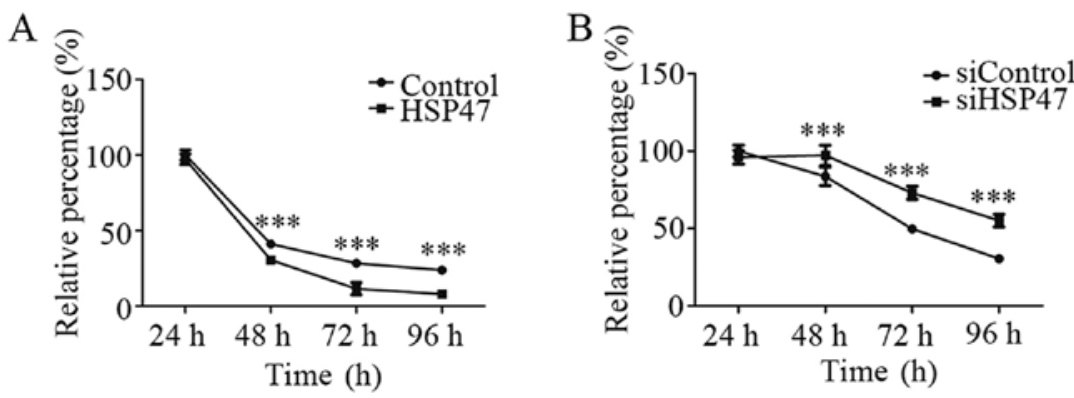

Figure 4. Influence of HSP47 overexpression or silencing on Hep-2 cell chemotherapy sensitivity. (A) HSP47 upregulation via plasmid vectors significantly increased the cell chemosensitivity to cisplatin. The cells were transfected with (pCDH)-HSP47 or siHSP47 for $24 \mathrm{~h}$, treated with cisplatin for $24-96 \mathrm{~h}$, and then subjected to cell proliferation assay by CCK-8. (B) HSP47 silencing via siRNA notably decreased the cell chemosensitivity to cisplatin; ${ }^{* * *} \mathrm{P}<0.001$.

transfection for 24 or $48 \mathrm{~h}, \mathrm{HSP} 47 \mathrm{mRNA}$ expression was significantly increased, by 10.3 and 4.6 times, respectively, in the (pCDH)-HSP47-transfected cells compared with the (pCDH)-control cells. In contrast, siHSP47-transfected cells respectively exhibited 68 and $69 \%$ lower expression than that noted in the siControl cells. The level of HSP47 protein expression was also detected by immunoblot analysis, which revealed an increase in the Hep-2-HSP47 cells (Fig. 2B) and a decrease in the Hep-2-siHSP47 cells (Fig. 2E).

Furthermore, we assessed the effect of HSP47 expression on cell viability using CCK-8 assay. Compared with the control, Hep-2 cells exhibited a marked reduction (Fig. 2C) in cell viability when HSP47 was upregulated, while there was a significant increase (Fig. 2F) when HSP47 was silenced. At $96 \mathrm{~h}$ after transfection, the cells with upregulation of HSP47 were
$19.3 \%$ less viable when compared with the $(\mathrm{pCDH})$-control cells. Meanwhile, HSP47-knockdown cells were $16.4 \%$ more viable than the siControl cells. The present study indicated that upregulation of HSP47 inhibited the growth of Hep-2 cells, whereas HSP47 downregulation stimulated proliferation.

HSP47 overexpression suppresses the migration and invasion abilities of the Hep-2 cells. We also investigated whether HSP47 upregulation inhibits cell migration and invasive abilities by wound-healing and transwell invasion assays, respectively. HSP47 upregulation markedly diminished wound-healing migration in the Hep-2 cells (Fig. 3A), and the ability to migrate was decreased by $20 \%$ (Fig. 3B; $\mathrm{P}<0.05$ ). Moreover, upregulation of HSP47 resulted in notably decreased migration (by 62.3\%) into the lower chamber in triplicate 

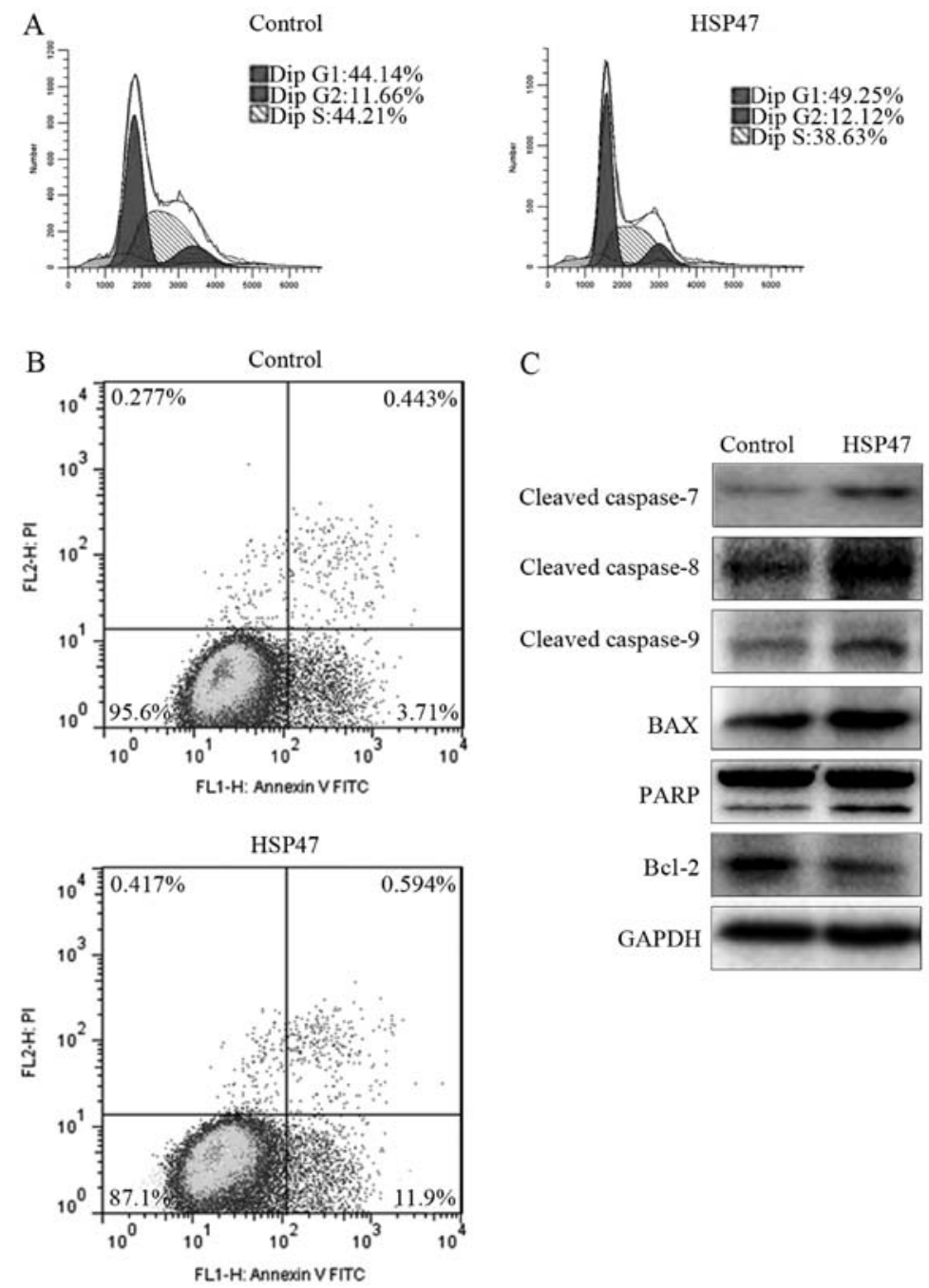

Figure 5. HSP47 upregulation promotes Hep-2 cell apoptosis and induces G1 phase arrest. (A) Cell cycle analysis showed that the G1 phase arrest in (pCDH)-HSP47-transfected cells was markedly increased compared with that in the (pCDH)-control cells. (B) Apoptosis assay showed that the percentage of early apoptotic cells was obviously increased in the HSP47-upregulated cells compared with the control. Annexin V FITC $/ \mathrm{PI}^{-}$, early apoptotic cells. (C) Western blot analysis showed that PARP, cleaved caspase-7/-8/-9 and Bax were markedly upregulated in (pCDH)-HSP47-transfected cells compared with levels in the (pCDH)-control cells, while Bcl-2 was notably downregulated.

independent assays (Fig. $3 \mathrm{C}$ and $\mathrm{D}$; $\mathrm{P}<0.001$ ). From these assays, we demonstrated that HSP47 upregulation suppressed Hep-2 cell mobility.

HSP47 modulates chemosensitivity to cisplatin in Hep-2 cells. We next evaluated the potential effect on sensitivity to chemotherapy by HSP47 in LSCC cells. As shown in Fig. 4A, upregulation of HSP47 significantly increased the cell chemosensitivity to cisplatin, by $25.9 \%$, at $48 \mathrm{~h}$. In contrast, knockdown of HSP47 led to more resistance to cisplatin chemotherapy, with a $16.2 \%$ increase compared with the negative-control group at $48 \mathrm{~h}$ (Fig. 4B). These results revealed that HSP47 overexpression sensitized the Hep-2 cell line to cisplatin chemotherapy, whereas HSP47 silencing protected cells against cisplatin.

HSP47 upregulation enhances the apoptosis of Hep-2 cells. To investigate the mechanisms underlying the growth inhibition and chemosensitivity enhancement noted in the HSP47-upregulated cells, we assessed cell cycle distribution and apoptosis by flow cytometric assay at $48 \mathrm{~h}$ after transfection. As shown in Fig. 5A, upregulation of HSP47 resulted in marked G1 phase arrest, with an increase of $11.6 \%$, compared with the cell cycle distribution of the control cells. This G1 phase arrest was accompanied by a $12.6 \%$ decrease in the percentage of $S$ phase cells. Meanwhile, the percentage of cells in the lower right quadrant, which represent early apoptotic cells, increased by $221 \%$ compared with the control (Fig. 5B). However, no obvious difference in late apoptotic cells or necrotic cells was noted. These findings indicated that overexpression of HSP47 mainly arrested Hep-2 cells in the G1 phase and promoted early apoptosis.

To further elucidate the molecules downstream of HSP47, the expression of apoptosis-related biomarkers was detected via western blotting, including PARP, cleaved caspase-7/-8/-9, Bcl-2 and Bax. As shown in Fig. 5C, the above-mentioned biomarkers were markedly upregulated in the (pCDH)-HSP47transfected cells compared with these levels noted in the (pCDH)-control cells, except for Bcl-2 protein, which was notably downregulated. 


\section{Discussion}

Overexpression of heat shock proteins (HSPs) has previously been demonstrated to be associated with poor prognosis in cancer patients, such as HSP70 overexpression in colorectal cancer (22), HSP27 overexpression in osteosarcoma (23) and breast cancer (24), HSP72 overexpression in renal cancer (25), and HSP90 overexpression in breast cancer (26). Moreover, malignant glioma patients with low expression of HSP73 had significantly longer progression-free survival than those with high HSP73 expression (27). In contrast, HSP27 overexpression was associated with a more favourable prognosis in malignant fibrous histiocytoma (28). These findings indicated that the prognostic signature of HSPs may be dependent on the cancer type. Our present study found that the low expression of HSP47 was significantly correlated with poor prognosis in LSCC patients by promoting Hep-2 cell proliferation and enhancing the resistance to cisplatin chemotherapy.

Previous research found that HSP47 was overexpressed in several human diseases and was highly related to tumourigenesis and poor prognosis $(15,29,30)$. However, subsequent research from multiple groups found that the expression of HSP47 is not always positively associated with unfavourable prognosis and progressive stages of disease. In particular, diabetic foot ulcer patients showed a significant decrease in HSP47 expression compared with wound patients without diabetes (31), and HSP47 inhibited the proliferation and collagen synthesis of keloid fibroblast cells (32). In the present study, we discovered that HSP47 protein expression in LSCC was markedly lower compared to that noted in the adjacent non-cancerous tissues by both immunoblot analysis and immunohistochemical staining. Moreover, an elevated tumour pathological grade of LSCC coupled with decreased expression of HSP47 protein was observed, and lower expression of HSP47 protein was associated with the poorer prognosis of the LSCC patients, indicating that HSP47 may act as a prognostic factor in LSCC. The cause of that outcome may be closely related to tissue-specific expression. HSP27 has been reported to be overexpressed and associated with poor prognosis in many human cancers, including breast, rectal, gastric, lung, liver and prostate cancers (33-36). Contrary to the findings in most cancers, in human esophageal squamous cell carcinoma (HESCC), the expression of HSP27 was low, and this low expression was correlated with poor prognosis $(37,38)$.

Furthermore, we assessed the biological significance of HSP47 in LSCC. We found that higher expression of HSP47 consistently resulted in inhibition of Hep-2 cell proliferation and promotion of sensitivity to cisplatin chemotherapy in vitro. In addition, upregulation of HSP47 expression also reduced the invasive ability, notably promoted the apoptosis of Hep-2 cells, and blocked the cells in the G1 phase. These observations indicated that HSP47 may be a potential therapeutic target in LSCC and indirectly supported the concept that low HSP47 expression is correlated with short survival and poor prognosis in LSCC. As shown in previous studies, HSP47 plays an important regulatory role in various types of tumours (39-41) and has a close relationship with tumour apoptosis $(40,42)$. However, the mechanism of HSP47-mediated Hep-2 cell apoptosis is still unknown. Previous research has indicated that apoptosis is regulated by changing the expression of a series of apoptosis-associated downstream genes, such as Bcl-2 family (proapoptotic Bax and antiapoptotic Bcl-2) and caspase family (caspase-7/-8/-9) proteins (43-45). As the above proteins are involved in tumour apoptosis, we also explored whether the levels of apoptosis-associated proteins were changed when HSP47 was upregulated in Hep-2 cells to further elucidate the molecules downstream of HSP47. The results showed that Bax was markedly increased in the (pCDH)-HSP47-transfected cells compared with that in the ( $\mathrm{pCDH})$-control cells, whereas Bcl-2 was decreased, indicating that HSP47 overexpression promoted tumour apoptosis. We speculate that upregulation of HSP47 may suppress the expression of Bcl-2, which plays a key role in apoptotic signal transduction through inhibition of intracellular signalling proteins and the expression of Bax (46-48). In accordance with the results for Bax/Bcl-2, HSP47 overexpression also resulted in increased expression of cleaved caspase-7/-8/-9 and PARP. Caspase- 8 and caspase-9 both play a role in the extrinsic apoptotic pathway and the intrinsic mitochondrial apoptotic pathway, respectively, given that both of them can activate downstream caspase family proteins, such as caspase-7 $(44,49,50)$. Therefore, it appears that HSP47 induces apoptosis via both the extrinsic and the intrinsic mitochondrial apoptosis pathways.

In conclusion, the present study demonstrated that there is a significant positive correlation between HSP47 and prognosis in LSCC patients, as the low expression of HSP47 was significantly correlated with poor prognosis. Moreover, in vitro, it was confirmed that HSP47 protein plays an important role in the biological behaviour of Hep-2 cells by inhibiting cell proliferation and invasion, enhancing sensitivity to cisplatin chemotherapy and promoting apoptosis. In brief, the present study revealed that HSP47 may be a potential prognostic biomarker and an attractive therapeutic target in LSCC. Further studies regarding the role of HSP47 expression in more LSCC patients may be worthwhile and the precise mechanism and regulatory pathways remain to be elucidated.

\section{Acknowledgements}

We thank the patients who participated in the present study, and Dr Guorong Chen, Fang Wang and Yi Jin for assistance in preparing the paraffin sections and immunohistochemistry analysis. This work was supported by funds from the National Natural Science Foundation of China (81201687), the Zhejiang Provincial Natural Science Foundation of China (LY14H130003, LY16H160051 and LY13H090009), and the Zhejiang Province Medical and Health Science and Technology Plan Project (2012RCA042).

\section{References}

1. Farhadieh RD, Rees CG, Yang JL, Salardini A, Russell P and Smee R: Radiotherapy in larynx squamous cell carcinoma is not associated with an increased diagnosis of second primary tumours. Clin Oncol (R Coll Radiol) 21: 315-319, 2009.

2. Yilmaz M, Karatas OF, Yuceturk B, Dag H, Yener M and Ozen M: Alpha-B-crystallin expression in human laryngeal squamous cell carcinoma tissues. Head Neck 37: 1344-1348, 2015.

3. Parkin DM, Bray F, Ferlay J and Pisani P: Global cancer statistics, 2002. CA Cancer J Clin 55: 74-108, 2005.

4. Jemal A, Siegel R, Ward E, Murray T, Xu J and Thun MJ: Cancer statistics, 2007. CA Cancer J Clin 57: 43-66, 2007. 
5. Tai $\mathrm{P}, \mathrm{Yu} \mathrm{E}$, Shiels $\mathrm{R}$ and Tonita J: Long-term survival rates of laryngeal cancer patients treated by radiation and surgery, radiation alone, and surgery alone: Studied by lognormal and Kaplan-Meier survival methods. BMC Cancer 5: 13, 2005.

6. Varghese BT, Sebastian P and Mathew A: Treatment outcome in patients undergoing surgery for carcinoma larynx and hypopharynx: A follow-up study. Acta Otolaryngol 129: 1480-1485, 2009 .

7. Ghaffar S, Akhtar S, Ikram M, Imam SZ and Sepah YJ: Comparison of different treatment modalities in advanced laryngeal hypopharyngeal squamous cell carcinoma. J Coll Physicians Surg Pak 20: 171-174, 2010.

8. Belcher R, Hayes K, Fedewa S and Chen AY: Current treatment of head and neck squamous cell cancer. J Surg Oncol 110: 551-574, 2014

9. Cooper JS, Zhang Q, Pajak TF, Forastiere AA, Jacobs J, Saxman SB, Kish JA, Kim HE, Cmelak AJ, Rotman M, et al: Long-term follow-up of the RTOG 9501/intergroup phase III trial: Postoperative concurrent radiation therapy and chemotherapy in high-risk squamous cell carcinoma of the head and neck. Int J Radiat Oncol Biol Phys 84: 1198-1205, 2012.

10. Sauk JJ, Nikitakis N and Siavash H: Hsp47 a novel collagen binding serpin chaperone, autoantigen and therapeutic target. Front Biosci 10: 107-118, 2005.

11. Bekri S, Adélaïde J, Merscher S, Grosgeorge J, Caroli-Bosc F, Perucca-Lostanlen D, Kelley PM, Pébusque MJ, Theillet C, Birnbaum D, et al: Detailed map of a region commonly amplified at 11q13-->q14 in human breast carcinoma. Cytogenet Cell Genet 79: 125-131, 1997.

12. Li D, Guang W, Abuzeid WM, Roy S, Gao GP, Sauk JJ and O'Malley BW Jr: Novel adenoviral gene delivery system targeted against head and neck cancer. Laryngoscope 118: 650-658, 2008.

13. Kwon YJ, Lee SJ, Koh JS, Kim SH, Kim YJ and Park JH: Expression patterns of aurora kinase B, heat shock protein 47 and periostin in esophageal squamous cell carcinoma. Oncol Res 18: 141-151, 2009.

14. Reese C, Lee R, Bonner M, Perry B, Heywood J, Silver RM, Tourkina E, Visconti RP and Hoffman S: Fibrocytes in the fibrotic lung: Altered phenotype detected by flow cytometry. Front Pharmacol 5: 141, 2014.

15. Zhao D, Jiang X, Yao C, Zhang L, Liu H, Xia H and Wang Y: Heat shock protein 47 regulated by miR-29a to enhance glioma tumor growth and invasion. J Neurooncol 118: 39-47, 2014.

16. Yamamoto N, Kinoshita T, Nohata N, Yoshino H, Itesako T, Fujimura L, Mitsuhashi A, Usui H, Enokida H, Nakagawa M, et al: Tumor-suppressive microRNA-29a inhibits cancer cell migration and invasion via targeting HSP47 in cervical squamous cell carcinoma. Int J Oncol 43: 1855-1863, 2013.

17. Araki K, Mikami T, Yoshida T, Kikuchi M, Sato Y, Oh-ishi M, Kodera Y, Maeda T and Okayasu I: High expression of HSP47 in ulcerative colitis-associated carcinomas: Proteomic approach. $\mathrm{Br}$ J Cancer 101: 492-497, 2009.

18. Chen HX and Cleck JN: Adverse effects of anticancer agents that target the VEGF pathway. Nat Rev Clin Oncol 6: 465-477, 2009

19. Tseng MY, Liu SY, Chen HR, Wu YJ, Chiu CC, Chan PT, Chiang WF, Liu YC, Lu CY, Jou YS, et al: Serine protease inhibitor (SERPIN) B1 promotes oral cancer cell motility and is over-expressed in invasive oral squamous cell carcinoma. Oral Oncol 45: 771-776, 2009.

20. Catzavelos C, Bhattacharya N, Ung YC, Wilson JA, Roncari L, Sandhu C, Shaw P, Yeger H, Morava-Protzner I, Kapusta L, et al: Decreased levels of the cell-cycle inhibitor p27Kip1 protein: Prognostic implications in primary breast cancer. Nat Med 3 : 227-230, 1997

21. Chappuis PO, Donato E, Goffin JR, Wong N, Bégin LR, Kapusta LR, Brunet JS, Porter P and Foulkes WD: Cyclin E expression in breast cancer: Predicting germline $B R C A 1$ mutations, prognosis and response to treatment. Ann Oncol 16 735-742, 2005.

22. Lazaris AC, Theodoropoulos GE, Davaris PS, Panoussopoulos D, Nakopoulou L, Kittas C and Golematis BC: Heat shock protein 70 and HLA-DR molecules tissue expression. Prognostic implications in colorectal cancer. Dis Colon Rectum 38: 739-745, 1995.

23. Uozaki $H$, Horiuchi $H$, Ishida $T$, Iijima $T$, Imamura $T$ and Machinami R: Overexpression of resistance-related proteins (metallothioneins, glutathione-S-transferase pi, heat shock protein 27, and lung resistance-related protein) in osteosarcoma. Relationship with poor prognosis. Cancer 79: 2336-2344, 1997.
24. Vargas-Roig LM, Gago FE, Tello O, Aznar JC and Ciocca DR: Heat shock protein expression and drug resistance in breast cancer patients treated with induction chemotherapy. Int $\mathrm{J}$ Cancer 79: 468-475, 1998 .

25. Santarosa M, Favaro D, Quaia M and Galligioni E: Expression of heat shock protein 72 in renal cell carcinoma: Possible role and prognostic implications in cancer patients. Eur J Cancer 33: 873-877, 1997.

26. Pick E, Kluger Y, Giltnane JM, Moeder C, Camp RL, Rimm DL and Kluger HM: High HSP90 expression is associated with decreased survival in breast cancer. Cancer Res 67: 2932-2937, 2007.

27. Hermisson M, Strik H, Rieger J, Dichgans J, Meyermann R and Weller M: Expression and functional activity of heat shock proteins in human glioblastoma multiforme. Neurology 54: 1357-1365, 2000.

28. Têtu B, Lacasse B, Bouchard HL, Lagacé R, Huot J and Landry J: Prognostic influence of HSP-27 expression in malignant fibrous histiocytoma: A clinicopathological and immunohistochemical study. Cancer Res 52: 2325-2328, 1992.

29. Lee HW, Kwon J, Kang MC, Noh MK, Koh JS, Kim JH and Park JH: Overexpression of HSP47 in esophageal squamous cell carcinoma: Clinical implications and functional analysis. Dis Esophagus 29: 848-855, 2016.

30. Zhu J, Xiong G, Fu H, Evers BM, Zhou BP and Xu R: Chaperone Hsp47 drives malignant growth and invasion by modulating an ECM gene network. Cancer Res 75: 1580-1591, 2015.

31. Singh K, Agrawal NK, Gupta SK, Mohan G, Chaturvedi S and Singh K: Decreased expression of heat shock proteins may lead to compromised wound healing in type 2 diabetes mellitus patients. J Diabetes Complications 29: 578-588, 2015.

32. Chen J, Zhao S, Liu Y, Cen Y and Nicolas C: Effect of captopril on collagen metabolisms in keloid fibroblast cells. ANZ J Surg 86: 1046-1051, 2016.

33. Kang SH, Kang KW, Kim KH, Kwon B, Kim SK, Lee HY, Kong SY, Lee ES, Jang SG and Yoo BC: Upregulated HSP27 in human breast cancer cells reduces Herceptin susceptibility by increasing Her2 protein stability. BMC Cancer 8: 286, 2008.

34. Tweedle EM, Khattak I, Ang CW, Nedjadi T, Jenkins R, Park BK, Kalirai H, Dodson A, Azadeh B, Terlizzo M, et al: Low molecular weight heat shock protein HSP27 is a prognostic indicator in rectal cancer but not colon cancer. Gut 59: 1501-1510, 2010.

35. Voll EA, Ogden IM, Pavese JM, Huang X, Xu L, Jovanovic BD and Bergan RC: Heat shock protein 27 regulates human prostate cancer cell motility and metastatic progression. Oncotarget 5: 2648-2663, 2014.

36. Ciocca DR and Calderwood SK: Heat shock proteins in cancer: Diagnostic, prognostic, predictive, and treatment implications. Cell Stress Chaperones 10: 86-103, 2005.

37. Xue L, Yang L, Jin ZA, Gao F, Kang JQ, Xu GH, Liu B, Li H, Wang XJ, Liu LJ, et al: Increased expression of HSP27 inhibits invasion and metastasis in human esophageal squamous cell carcinoma. Tumour Biol 35: 6999-7007, 2014.

38. Langer R, Ott K, Specht K, Becker K, Lordick F, Burian M, Herrmann K, Schrattenholz A, Cahill MA, Schwaiger M, et al: Protein expression profiling in esophageal adenocarcinoma patients indicates association of heat-shock protein 27 expression and chemotherapy response. Clin Cancer Res 14: 8279-8287, 2008.

39. Yohannes E, Chang J, Tar MT, Davies KP and Chance MR: Molecular targets for diabetes mellitus-associated erectile dysfunction. Mol Cell Proteomics 9: 565-578, 2010.

40. Kawasaki K, Ushioda R, Ito S, Ikeda K, Masago Y and Nagata K: Deletion of the collagen-specific molecular chaperone Hsp47 causes endoplasmic reticulum stress-mediated apoptosis of hepatic stellate cells. J Biol Chem 290: 3639-3646, 2015.

41. Miyata S, Mizuno T, Koyama Y, Katayama T and Tohyama M: The endoplasmic reticulum-resident chaperone heat shock protein 47 protects the Golgi apparatus from the effects of $O$-glycosylation inhibition. PLoS One 8: e69732, 2013.

42. Zeng YL, Zhang XJ, Shang J, Ding GQ and Kang Y: Single-chain human anti-EGFR antibody/truncated protamine fusion protein carrying Hsp47 siRNA can induce apoptosis of human hepatic stellate cells. Zhonghua Gan Zang Bing Za Zhi 22: 843-848, 2014 (In Chinese)

43. Pećina-Slaus N: Wnt signal transduction pathway and apoptosis: A review. Cancer Cell Int 10: 22, 2010. 
44. Fulda S and Debatin KM: Extrinsic versus intrinsic apoptosis pathways in anticancer chemotherapy. Oncogene 25: 4798-4811, 2006.

45. Faião-Flores F, Coelho PR, Toledo Arruda-Neto JD, Maria-Engler SS, Tiago M, Capelozzi VL, Giorgi RR and Maria DA: Apoptosis through $\mathrm{Bcl}-2 / \mathrm{Bax}$ and cleaved caspase up-regulation in melanoma treated by boron neutron capture therapy. PLoS One 8: e59639, 2013.

46. Kiefer MC, Brauer MJ, Powers VC, Wu JJ, Umansky SR, Tomei LD and Barr PJ: Modulation of apoptosis by the widely distributed Bcl-2 homologue Bak. Nature 374: 736-739, 1995.

47. Chung TK, Cheung TH, Lo WK, Yim SF, Yu MY, Krajewski S, Reed JC and Wong YF: Expression of apoptotic regulators and their significance in cervical cancer. Cancer Lett 180: 63-68, 2002.
48. Sharma H, Sen S, Mathur M, Bahadur S and Singh N: Combined evaluation of expression of telomerase, survivin, and anti-apoptotic Bcl-2 family members in relation to loss of differentiation and apoptosis in human head and neck cancers. Head Neck 26: 733-740, 2004.

49. Kleinberg L and Davidson B: Cell survival and apoptosis-related molecules in cancer cells in effusions: A comprehensive review. Diagn Cytopathol 37: 613-624, 2009.

50. Olsson M and Zhivotovsky B: Caspases and cancer. Cell Death Differ 18: 1441-1449, 2011. 